Article

\title{
Synthesis and Positive Inotropic Activity of $[1,2,4]$ Triazolo[4,3-a] Quinoxaline Derivatives Bearing Substituted Benzylpiperazine and Benzoylpiperazine Moieties
}

\author{
Xue-Kun Liu ${ }^{1,+}{ }^{\text {, Long-Xu Ma }}{ }^{2,+}{ }^{\dagger}$ Zhi-Yu Wei ${ }^{2}$, Xun Cui ${ }^{3}$, Shi Zhan ${ }^{4}$, Xiu-Mei Yin ${ }^{2, *}$ \\ and Hu-Ri Piao ${ }^{2, *}$ \\ 1 Tonghua Normal University , College of Pharmaceutical and Food Science, Tonghua 134002, China; \\ liuxuekunth@126.com \\ 2 Key Laboratory of Natural Resources of Changbai Mountain \& Functional Molecules, Ministry of Education, \\ College of Pharmacy, Yanbian University, Yanji 133000, China; xuekunliu@sina.com (L.-X.M.); \\ 15981392912@163.com (Z.-Y.W.) \\ 3 College of Medicine, Yanbian University, Yanji 133000, China; cuixun@ybu.edu.cn \\ 4 State Key Laboratory of Inorganic Synthesis and Preparative Chemistry, College of Chemistry, \\ Jilin University, Changchun 130000, China; yqwang@126.com \\ * Correspondences: xmyin@ybu.edu.cn (X.-M.Y.); piaohr@ybu.edu.cn (H.-R.P.); \\ Tel.: +86-433-243-6019 (X.-M.Y.); +86-433-243-5003 (H.-R.P.); \\ Fax: +86-433-243-5026 (X.-M.Y.); +86-433-243-5026 (H.-R.P.) \\ + These authors contributed equally to this work.
}

Academic Editor: Derek J. McPhee

Received: 15 January 2017; Accepted: 8 February 2017; Published: 11 February 2017

\begin{abstract}
In an attempt to search for more potent positive inotropic agents, two series of $[1,2,4]$ triazolo[4,3- $a]$ quinoxaline derivatives bearing substituted benzylpiperazine and benzoylpiperazine moieties were synthesized and their positive inotropic activities evaluated by measuring left atrial stroke volume in isolated rabbit heart preparations. Several compounds showed favorable activities compared with the standard drug, milrinone. Compound $\mathbf{6} \mathbf{c}$ was the most potent agent, with an increased stroke volume of $12.53 \% \pm 0.30 \%$ (milrinone: $2.46 \% \pm 0.07 \%$ ) at $3 \times 10^{-5} \mathrm{M}$. The chronotropic effects of compounds having considerable inotropic effects were also evaluated.
\end{abstract}

Keywords: $[1,2,4]$ triazolo[4,3-a] quinoxaline; positive inotropic activity; stroke volume; atrium; milrinone

\section{Introduction}

Glycosides such as digoxin are frequently prescribed cardiotonic agents used for the treatment of congestive heart failure (CHF). Unlike other CHF drugs, they do not increase mortality. However, the narrow safety margins associated with the use of digitalis compounds are serious problems because of the high frequency and severity of digitalis intoxication [1]. The discovery of amrinone led to the synthesis of several agents holding promise for CHF treatment as non-sympathomimetic, non-glycoside agents [2]. The phosphodiesterase-inhibiting agent milrinone has vasodilator and inotropic properties. It was approved for the treatment of CHF more than a decade ago. Nevertheless, the significant ventricular arrhythmias and tachycardia associated with elevated levels of cyclic adenosine monophosphate limit the use of milrinone [3] as well as a newer agent, vesnarinone [4,5]. Therefore, newer positive inotropic agents with fewer side effects are needed [6].

Previously, we reported the identification of a $[1,2,4]$ triazolo[ $[3,4-a]$ phthalazine derivative (compound $\mathbf{A}$ ) with remarkable positive inotropic activities that could elicit an increased stroke 
volume of $9.92 \% \pm 0.09 \%$ (Figure 1) [7]. To further optimize compound $\mathbf{A}$, based on the principles of bioisosterism, we replaced the $[1,2,4]$ triazolo[3,4- $a]$ phthalazine group with a $[1,2,4]$ triazolo[4,3- $a]$ quinoxaline group, and simultaneously changed the substituents on the phenyl ring of the benzyl moiety at the 4-position of the piperazine ring, Thus, 11 novel compounds of $[1,2,4]$ triazolo[4,3-a] quinoxaline derivatives bearing substituted benzylpiperazine moieties (6a-k) were synthesized. Moreover, to investigate the effects of benzylpiperazine on activity, we introduced a carbonyl group at the $\alpha$-position of the benzyl group. Thus, a series of $[1,2,4]$ triazolo[4,3- $a]$ quinoxaline derivatives bearing substituted benzoylpiperazine moieties $(7 \mathbf{a}-\mathbf{e})$ were also designed and screened for their inotropic activity.<smiles>Cc1[nH]c(=O)c(C#N)cc1-c1ccncc1</smiles>

Milrinone<smiles>COc1ccc(C(=O)N2CCN(c3ccc4c(c3)CCC(=O)N4)CC2)cc1OC</smiles><smiles></smiles>

compound 1

Figure 1. Cardiotonic agents used for the treatment of congestive heart failure $(\mathrm{CHF})$ and the previously reported compound $\mathbf{A}$.

\section{Results and Discussion}

\subsection{Synthesis}

The reaction sequence for the synthesis of $\mathbf{1 6}$ new quinoxaline derivatives $\mathbf{6 a}-\mathbf{k}$ and $7 \mathbf{a}-\mathbf{e}$ is outlined in Scheme 1. Benzene-1,2-diamine (1) was reacted with diethyl oxalate to afford quinoxaline$2,3(1 H, 4 H)$-dione (2), which was subsequently reacted with refluxing hydrazine hydrate to give 3-hydrazono-3,4-dihydroquinoxalin-2(1H)-one (3). Compound 4 was prepared from compound 3 by reaction with ethyl orthoformate. Compound 5 was obtained by reacting 4 with refluxing phosphorus oxychloride $\left(\mathrm{POCl}_{3}\right)$. The nucleophilic aromatic substitution reactions of 5 with various monosubstituted piperazines in refluxing acetone in the presence of potassium carbonate afforded compounds $\mathbf{6 a - k}$. Finally, compounds $7 \mathbf{a}-\mathbf{e}$ were obtained in high yield from the reactions of $\mathbf{5}$ with appropriate different monosubstituted piperazines in refluxing acetone in the presence of potassium carbonate. Newly synthesized derivatives $\mathbf{6 a}-\mathbf{k}$ and $\mathbf{7 a}-\mathbf{e}$ were characterized by ${ }^{1} \mathrm{H}-\mathrm{NMR}$, ${ }^{13} \mathrm{C}-\mathrm{NMR}$, IR and mass spectral data. In general, IR spectral data in the ranges of $1549-1457 \mathrm{~cm}^{-1}$ and $1638-1620 \mathrm{~cm}^{-1}$ indicated distinctive functional groups such as $-\mathrm{C}=\mathrm{N}$ and $-\mathrm{C}=\mathrm{O}$ stand for derivatives $\mathbf{6 a}-\mathbf{k}$ and $\mathbf{7 a}-\mathbf{e}$, respectively. The $(\mathrm{M}+1)$ peaks in the mass spectra for these compounds were in agreement with their molecular formula. 
<smiles>Cc1ccc2[nH]c(=O)c(=O)[nH]c2c1</smiles>

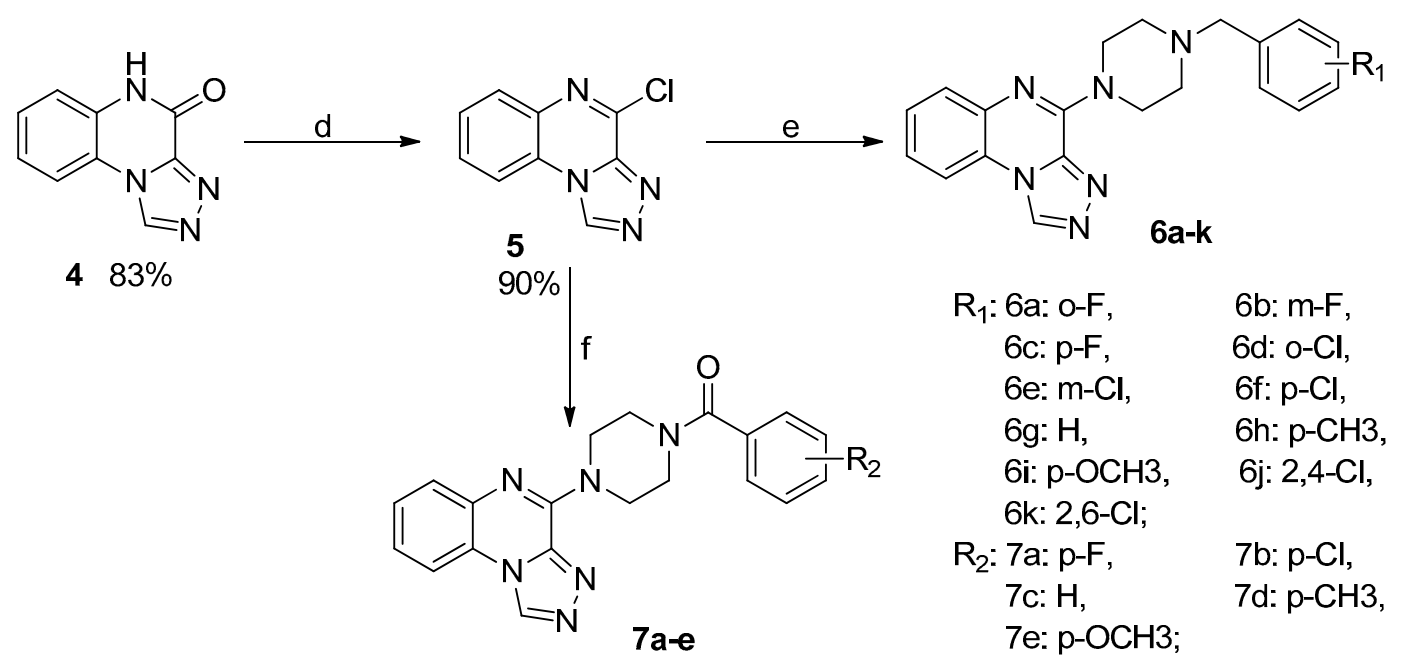

Scheme 1. Synthetic scheme for the synthesis of compound $\mathbf{6 a}-\mathbf{k}$ and $7 \mathbf{a}-\mathbf{e}$. Reagents and conditions: (a) $\mathrm{C}_{6} \mathrm{H}_{10} \mathrm{O}_{4}$, reflux, $4 \mathrm{~h}$; (b) $\mathrm{NH}_{2} \mathrm{NH}_{2} \mathrm{H}_{2} \mathrm{O}$, reflux, $4 \mathrm{~h}$; (c) $\mathrm{CH}\left(\mathrm{OC}_{2} \mathrm{H}_{5}\right)_{3}$, reflux, $3 \mathrm{~h}$; (d) $\mathrm{POCl}_{3}$, Methylbenzene/DMF, reflux, $3 \mathrm{~h}$; (e) monosubstituted piperazines, K2CO3, acetone, reflux, $5 \mathrm{~h}$; (f) monosubstituted piperazines, $\mathrm{K}_{2} \mathrm{CO}_{3}$, acetone, reflux, $8 \mathrm{~h}$.

\subsection{Biological Evaluation}

Seven of the 16 compounds tested displayed inotropic effects against isolated rabbit heart preparations (Table 1 ). Compounds $\mathbf{6 c}, \mathbf{6 g}, \mathbf{6} \mathbf{h}$, and $\mathbf{7 c}$ exhibited more potent effects compared with milrinone $\left(2.46 \% \pm 0.3 \%\right.$ at $\left.3 \times 10^{-5} \mathrm{M}\right)$, among which compound $6 \mathrm{c}$ showed the most potent activity, with an increased stroke volume of $12.53 \% \pm 0.30 \%$. For compounds $6 \mathbf{a}-\mathbf{k}$, different substituents on the phenyl ring of the benzyl group at the 4-position of the piperazine ring exerted considerable influence on the inotropic activity. For fluorinated compounds, only para-substituted $\mathbf{6 c}$ showed good activity, and clearly exhibited more potent effects compared with lead compound $\mathbf{1}$ and milrinone, with an increased stroke volume of $12.53 \% \pm 0.30 \%$. Chloro-substituted compounds $(\mathbf{6 d}, \mathbf{6 e}, \mathbf{6 j}$ and $\mathbf{6 k})$ did not show any inotropic activity, and the para-chloro-substituted 6 f displayed slightly increased activity with an increased stroke volume of $1.01 \% \pm 0.06 \%$. The position of the substituents on the phenyl ring also influenced activity but, in general, a clear pattern for the structure-activity relationship was not found.

In the series of [4-([1,2,4]triazolo[4,3-a]quinoxalin-4-yl)piperazin-1-yl](phenyl)methanone-bearing substituted benzoylpiperazine moieties (7a-e), compounds possessing the same substituent group as series $\mathbf{6}$ showed lower activity than those in series $\mathbf{6}$. Compound 7 a displayed slightly increased activity with an increased stroke volume of $0.99 \% \pm 0.06 \%$. Only $7 \mathrm{c}$ exhibited more potent effects compared with milrinone, and increased the stroke volume by $4.71 \% \pm 0.05 \%$.

We investigated the dynamics of the tested compounds in perfused beating rabbit atria. Compounds $6 \mathrm{c}, 6 \mathrm{~g}$ and $6 \mathrm{~h}$ produced initial increases in stroke volume, whereas longer treatment caused decreases in stroke volume (Figure $2 \mathrm{~A}-\mathrm{C}$ ). For compound $\mathbf{7 c}$, the stroke volume increased gradually (Figure 2D).

Next, we tested the dose dependency of the most effective compound (6c) at $1 \times 10^{-5} \mathrm{M}$, $3 \times 10^{-5} \mathrm{M}$ and $1 \times 10^{-4} \mathrm{M}$. This compound showed maximal effects at $3 \times 10^{-5} \mathrm{M}$, and lower activity at the highest dose $\left(1 \times 10^{-4} \mathrm{M}\right)$ (Figure 3 ). 
Table 1. Positive inotropic activity of the test compounds.

\begin{tabular}{|c|c|c|}
\hline & $a-k$ & $7 a e$ \\
\hline Compound & $\mathbf{R}$ & Increased Stroke Volume (\%) ${ }^{a}$ \\
\hline $6 a$ & $o-\mathrm{F}$ & $-\mathrm{b}$ \\
\hline $6 b$ & $m-\mathrm{F}$ & - \\
\hline $6 c$ & $p-\mathrm{F}$ & $12.53 \pm 0.30$ \\
\hline $6 \mathrm{~d}$ & $o-\mathrm{Cl}$ & - \\
\hline $6 e$ & $m-\mathrm{Cl}$ & - \\
\hline $6 f$ & $p-\mathrm{Cl}$ & $1.01 \pm 0.06$ \\
\hline $6 \mathrm{~g}$ & $\mathrm{H}$ & $6.36 \pm 0.13$ \\
\hline $6 h$ & $p-\mathrm{CH}_{3}$ & $3.50 \pm 0.03$ \\
\hline $6 \mathbf{i}$ & $p-\mathrm{OCH}_{3}$ & $0.63 \pm 0.05$ \\
\hline $6 \mathbf{j}$ & $2,4-\mathrm{Cl}$ & - \\
\hline $6 \mathrm{k}$ & $2,6-\mathrm{Cl}$ & - \\
\hline $7 a$ & $p-\mathrm{F}$ & $0.99 \pm 0.06$ \\
\hline $7 \mathrm{~b}$ & $p-\mathrm{Cl}$ & - \\
\hline $7 \mathrm{c}$ & $\mathrm{H}$ & $4.71 \pm 0.05$ \\
\hline $7 d$ & $p-\mathrm{CH}_{3}$ & - \\
\hline A & - & $9.92 \pm 0.09$ \\
\hline milrinone & & $2.46 \pm 0.07$ \\
\hline
\end{tabular}

a The concentration for the test sample is $3 \times 10^{-5} \mathrm{M} .{ }^{\mathrm{b}}$ None or negative stroke volume increase.
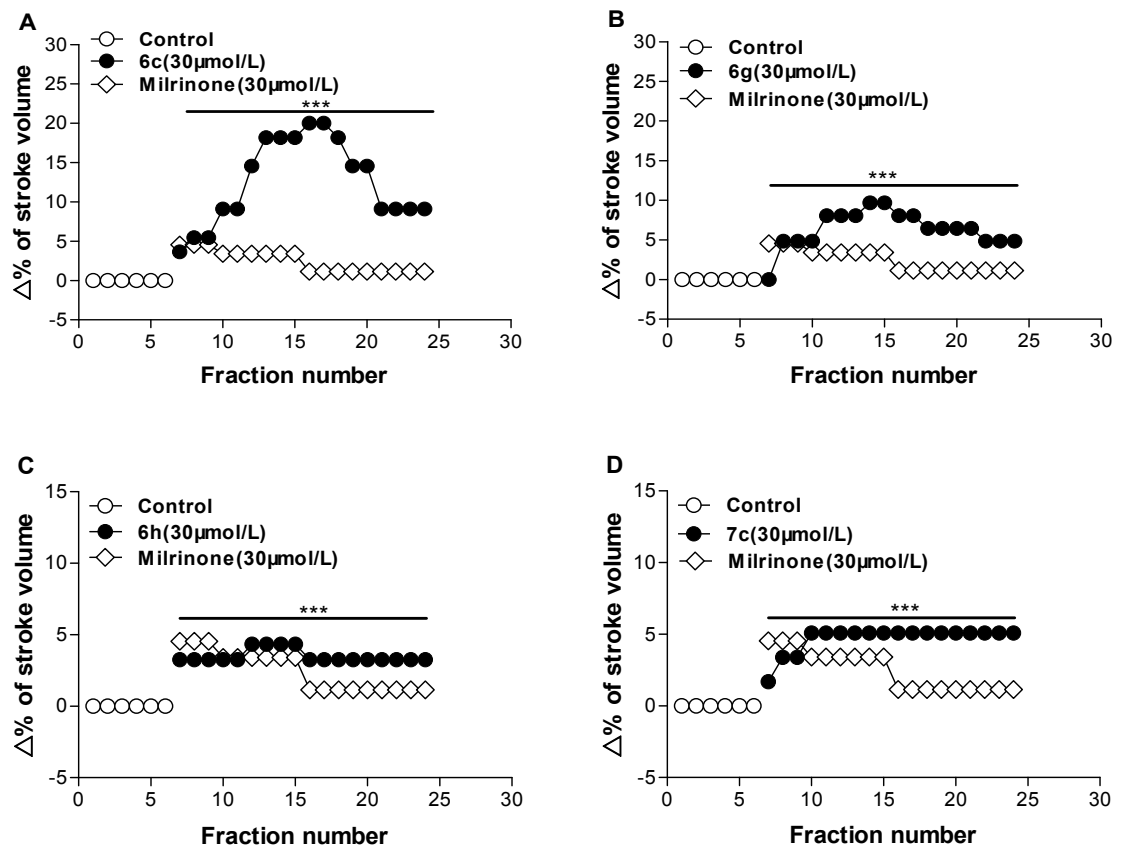

Figure 2. Effects of milrinone and compounds $6 \mathrm{c}, 6 \mathrm{~g}, 6 \mathrm{~h}$, and $7 \mathrm{c}$ on stroke volume in beating rabbit atria $(1.5 \mathrm{~Hz})$. Values are means \pm SE. ${ }^{* * *} p<0.001$ vs. control. (A): effects of compound $\mathbf{6 c}$ on stroke volume in beating rabbit atria, (B): effects of compound $6 \mathrm{~g}$ on stroke volume in beating rabbit atria, (C): effects of compound $\mathbf{6 h}$ on stroke volume in beating rabbit atria, (D): effects of compound 7c on stroke volume in beating rabbit atria. 


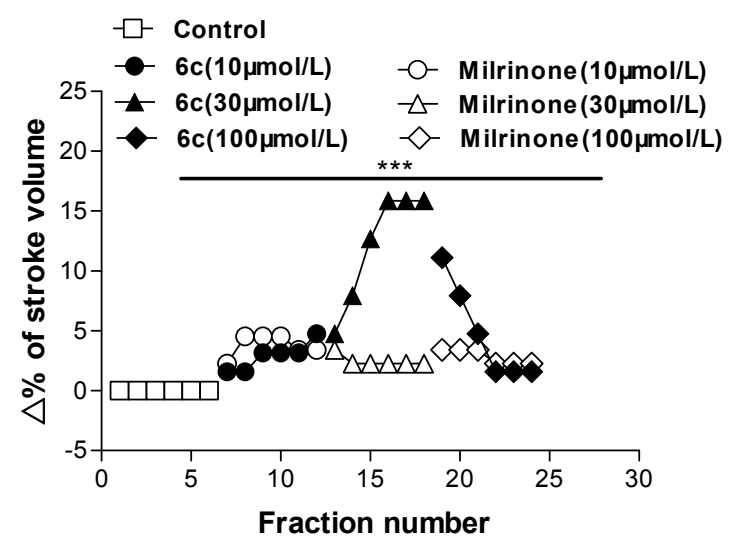

Figure 3. Concentration-response curves of compounds $6 \mathrm{c}$ and milrinone on stroke volume in beating rabbit atria $(1.5 \mathrm{~Hz})$. Values are means $\pm \mathrm{SE} .{ }^{* * *} p<0.001$ versus control.

\section{Experimental Section}

\subsection{General Information}

Melting points were determined in open capillary tubes and were uncorrected. Chemical reactions were monitored by thin-layer chromatography on silica gel precoated F254 plates (Merck, Whitehouse Station, NJ, USA). Developed plates were visualized by ultraviolet light $(254 \mathrm{~nm})$. Column chromatography was undertaken with 200-mesh silica gel (Merck). IR spectra were recorded (in KBr) on a FT-IR 1730 system. ${ }^{1} \mathrm{H}-\mathrm{NMR}$ spectra were measured on an AV-300 Spectrometer (Bruker, Billerica, MA, USA) using trimethylsilane as an internal standard. Mass spectra were measured on an HP1100LC system (Agilent Technologies, Santa Clara, CA, USA). Chemicals were purchased from Sigma-Aldrich (Saint Louis, MO, USA) and Fluka (Milwaukee, WI, USA).

\subsection{Synthesis}

3.2.1. General Experimental Procedure for the Synthesis of $[1,2,4]$ Triazolo[4,3- $a]$ Quinoxaline-Bearing Substituted Benzylpiperazine Moieties (6a-k)

Compounds 1-5 were synthesized by the previously described method [8-12]. A mixture of $5(0.20 \mathrm{~g}, 1.0 \mathrm{mmol})$, monosubstituted piperazine $(2.0 \mathrm{mmol})$ and anhydrous potassium carbonate in acetone was heated at reflux with stirring for $5 \mathrm{~h}$. The solvent was evaporated under reduced pressure, and the resulting residue dissolved in dichloromethane (DCM). The DCM solution was washed sequentially with water and brine, dried over $\mathrm{MgSO}_{4}$, and distilled to dryness under reduced pressure. The resulting residue was purified by silica gel column chromatography with DCM and methanol (30:1). The yield, melting point, and spectral data of each compound were recorded.

4-(4-(2-Fluorobenzyl)piperazin-1-yl)-[1,2,4]triazolo[4,3-a] quinoxaline (6a). Yield: 83\%; m.p. $160-164{ }^{\circ} \mathrm{C}$. IR $(\mathrm{KBr}) \mathrm{cm}^{-1}: 1542,1508,1474(\mathrm{C}=\mathrm{N}) .{ }^{1} \mathrm{H}-\mathrm{NMR}\left(\mathrm{CDCl}_{3}, 300 \mathrm{MHz}, \mathrm{ppm}\right): 2.62-2.63\left(\mathrm{~m}, 4 \mathrm{H},\left(\mathrm{CH}_{2}\right)_{2}\right)$, 3.49-3.55 (m, 4H, $\left.\left(\mathrm{CH}_{2}\right)_{2}\right), 4.46\left(\mathrm{~s}, 2 \mathrm{H}, \mathrm{CH}_{2}\right), 7.27-7.73(\mathrm{~m}, 8 \mathrm{H}, \mathrm{Ar}-\mathrm{H}), 9.17(\mathrm{~s}, 1 \mathrm{H}, \mathrm{N}=\mathrm{CH}) .{ }^{13} \mathrm{C}-\mathrm{NMR}$ $\left(\mathrm{CDCl}_{3}, 75 \mathrm{MHz}\right.$, ppm): $\delta 163.08,159.82,147.38,142.34,138.56,135.98,131.68,129.05,127.90,126.92$, $123.94,123.65,121.60,115.20,114.93,55.31,52.98,46.19 ; \mathrm{MS} m / z: 363(\mathrm{M}+1)$.

4-(4-(3-Fluorobenzyl)piperazin-1-yl)-[1,2,4]triazolo [4,3-a]quinoxaline (6b). Yield: 76\%; m.p. $146-150{ }^{\circ} \mathrm{C}$. IR (KBr) cm ${ }^{-1}: 1543,1512,1483(\mathrm{C}=\mathrm{N}) .{ }^{1} \mathrm{H}-\mathrm{NMR}\left(\mathrm{CDCl}_{3}, 300 \mathrm{MHz}, \mathrm{ppm}\right): 2.61-2.64\left(\mathrm{~m}, 4 \mathrm{H},\left(\mathrm{CH}_{2}\right)_{2}\right)$, 3.47-3.55 (m, 4H, $\left.\left(\mathrm{CH}_{2}\right)_{2}\right), 4.44\left(\mathrm{~s}, 2 \mathrm{H}, \mathrm{CH}_{2}\right), 6.95-7.69(\mathrm{~m}, 8 \mathrm{H}, \mathrm{Ar}-\mathrm{H}), 9.15(\mathrm{~s}, 1 \mathrm{H}, \mathrm{N}=\mathrm{CH}) .{ }^{13} \mathrm{C}-\mathrm{NMR}$ $\left(\mathrm{CDCl}_{3}, 75 \mathrm{MHz}, \mathrm{ppm}\right): \delta 164.60,161.35,145.67,140.59,139.92,136.90,135.52,129.67,127.90,126.94$, 124.60, 123.70, 121.62, 115.91, 115.63, 62.43, 53.24, 46.20; MS m/z: $363(\mathrm{M}+1)$. 
4-(4-(4-Fluorobenzyl)piperazin-1-yl)-[1,2,4]triazolo[4,3-a] quinoxaline (6c). Yield: 71\%; m.p. 156-158 ${ }^{\circ} \mathrm{C}$. IR (KBr) cm ${ }^{-1}: 1541,1512,1485(\mathrm{C}=\mathrm{N}) .{ }^{1} \mathrm{H}-\mathrm{NMR}\left(\mathrm{CDCl}_{3}, 300 \mathrm{MHz}, \mathrm{ppm}\right): 2.61-2.65\left(\mathrm{~m}, 4 \mathrm{H},\left(\mathrm{CH}_{2}\right)_{2}\right)$, 3.49-3.55 (m, 4H, $\left.\left(\mathrm{CH}_{2}\right)_{2}\right), 4.46\left(\mathrm{~s}, 2 \mathrm{H}, \mathrm{CH}_{2}\right), 7.00-7.73(\mathrm{~m}, 8 \mathrm{H}, \mathrm{Ar}-\mathrm{H}), 9.17(\mathrm{~s}, 1 \mathrm{H}, \mathrm{N}=\mathrm{CH}) .{ }^{13} \mathrm{C}-\mathrm{NMR}$ $\left(\mathrm{CDCl}_{3}, 75 \mathrm{MHz}, \mathrm{ppm}\right): \delta 145.73,139.90,138.08,137.03,135.40,133.63,130.75,129.03,127.90,127.00$, 124.62, 121.60, 114.65, 63.43, 52.91, 46.19; MS m/z: $363(\mathrm{M}+1)$.

4-(4-(2-Chlorobenzyl)piperazin-1-yl)-[1,2,4]triazolo[4,3-a] quinoxaline (6d). Yield: 72\%; m.p. $178-180{ }^{\circ} \mathrm{C}$. IR $(\mathrm{KBr}) \mathrm{cm}^{-1}: 1549,1508,1479(\mathrm{C}=\mathrm{N}) .{ }^{1} \mathrm{H}-\mathrm{NMR}\left(\mathrm{CDCl}_{3}, 300 \mathrm{MHz}, \mathrm{ppm}\right): 2.72-2.75\left(\mathrm{~m}, 4 \mathrm{H},\left(\mathrm{CH}_{2}\right)_{2}\right)$, 3.43-3.72 (m, 4H, $\left.\left(\mathrm{CH}_{2}\right)_{2}\right), 4.47\left(\mathrm{~s}, 2 \mathrm{H}, \mathrm{CH}_{2}\right), 7.22-7.74(\mathrm{~m}, 8 \mathrm{H}, \mathrm{Ar}-\mathrm{H}), 9.20(\mathrm{~s}, 1 \mathrm{H}, \mathrm{N}=\mathrm{CH}) .{ }^{13} \mathrm{C}-\mathrm{NMR}$ $\left(\mathrm{CDCl}_{3}, 75 \mathrm{MHz}, \mathrm{ppm}\right): \delta 145.69,139.94,136.91,135.56,135.28,134.47,130.93,129.55,128.40,128.00$, 126.95, 126.68, 122.72, 121.60, 111.52, 53.23, 50.66, 46.21; MS m/z: 379 (M + 1).

4-(4-(3-Chlorobenzyl)piperazin-1-yl)-[1,2,4]triazolo[4,3-a] quinoxaline (6e). Yield: 61\%; m.p. $160-162{ }^{\circ} \mathrm{C}$. IR (KBr) cm ${ }^{-1}: 1548,1511,1474(\mathrm{C}=\mathrm{N}) .{ }^{1} \mathrm{H}-\mathrm{NMR}\left(\mathrm{CDCl}_{3}, 300 \mathrm{MHz}, \mathrm{ppm}\right): 2.62-2.65\left(\mathrm{~m}, 4 \mathrm{H},\left(\mathrm{CH}_{2}\right)_{2}\right)$, 3.47-3.54 (m, 4H, $\left.\left(\mathrm{CH}_{2}\right)_{2}\right), 4.45\left(\mathrm{~s}, 2 \mathrm{H}, \mathrm{CH}_{2}\right), 7.19-7.71(\mathrm{~m}, 8 \mathrm{H}, \mathrm{Ar}-\mathrm{H}), 9.17(\mathrm{~s}, 1 \mathrm{H}, \mathrm{N}=\mathrm{CH}) .{ }^{13} \mathrm{C}-\mathrm{NMR}$ $\left(\mathrm{CDCl}_{3}, 75 \mathrm{MHz}, \mathrm{ppm}\right): \delta 145.66,140.94,139.96,136.88,135.55,134.25,134.13,129.59,129.47,129.12$, 127.94, 127.43, 126.95, 123.76, 121.61, 52.91, 50.60, 46.17; MS m/z: 379 (M + 1).

4-(4-(4-Chlorobenzyl)piperazin-1-yl)-[1,2,4]triazolo[4,3-a] quinoxaline (6f). Yield: 80\%; m.p. 162-164 ${ }^{\circ} \mathrm{C}$. IR (KBr) cm ${ }^{-1}: 1541,1512,1485(\mathrm{C}=\mathrm{N}) .{ }^{1} \mathrm{H}-\mathrm{NMR}\left(\mathrm{CDCl}_{3}, 300 \mathrm{MHz}, \mathrm{ppm}\right): 2.62-2.65\left(\mathrm{~m}, 4 \mathrm{H},\left(\mathrm{CH}_{2}\right)_{2}\right)$, 3.49-3.55 (m, 4H, $\left.\left(\mathrm{CH}_{2}\right)_{2}\right), 4.46\left(\mathrm{~s}, 2 \mathrm{H}, \mathrm{CH}_{2}\right), 7.27-7.73(\mathrm{~m}, 8 \mathrm{H}, \mathrm{Ar}-\mathrm{H}), 9.17(\mathrm{~s}, 1 \mathrm{H}, \mathrm{N}=\mathrm{CH}) .{ }^{13} \mathrm{C}-\mathrm{NMR}$ $\left(\mathrm{CDCl}_{3}, 75 \mathrm{MHz}, \mathrm{ppm}\right): \delta 145.70,139.96,137.28,136.93,135.50,133.00,130.75,129.03,127.97,127.00$, 124.26, 121.65, 114.50, 63.46, 53.19, 46.16; MS m/z: $379(\mathrm{M}+1)$.

4-(4-Benzylpiperazin-1-yl)-[1,2,4]triazolo[4,3-a] quinoxaline (6g). Yield: 89\%; m.p. $150-152{ }^{\circ} \mathrm{C}$. IR (KBr) $\mathrm{cm}^{-1}: 1548,1517,1467(\mathrm{C}=\mathrm{N}) .{ }^{1} \mathrm{H}-\mathrm{NMR}\left(\mathrm{CDCl}_{3}, 300 \mathrm{MHz}, \mathrm{ppm}\right): 2.63-2.66\left(\mathrm{~m}, 4 \mathrm{H},\left(\mathrm{CH}_{2}\right)_{2}\right), 3.48-3.58$ $\left(\mathrm{m}, 4 \mathrm{H},\left(\mathrm{CH}_{2}\right)_{2}\right), 4.46\left(\mathrm{~s}, 2 \mathrm{H}, \mathrm{CH}_{2}\right), 7.26-7.70(\mathrm{~m}, 8 \mathrm{H}, \mathrm{Ar}-\mathrm{H}), 9.15(\mathrm{~s}, 1 \mathrm{H}, \mathrm{N}=\mathrm{CH}) .{ }^{13} \mathrm{C}-\mathrm{NMR}\left(\mathrm{CDCl}_{3}\right.$, $75 \mathrm{MHz}, \mathrm{ppm}): \delta 145.71,139.85,137.71,136.98,135.50,129.26,128.21,128.11,127.24,126.97,123.67$, $121.62,114.48,63.08,53.27,46.24 ; \mathrm{MS} m / z: 345(\mathrm{M}+1)$.

4-(4-(4-Methylbenzyl)piperazin-1-yl)-[1,2,4]triazolo[4,3-a] quinoxaline (6h). Yield: 76\%; m.p. $156-158{ }^{\circ} \mathrm{C}$. IR (KBr) cm ${ }^{-1}: 1549,1506,1483(\mathrm{C}=\mathrm{N}) .{ }^{1} \mathrm{H}-\mathrm{NMR}\left(\mathrm{CDCl}_{3}, 300 \mathrm{MHz}, \mathrm{ppm}\right): 2.32-2.35\left(\mathrm{~m}, 3 \mathrm{H}, \mathrm{CH}_{3}\right)$, 2.61-2.74 (m, 4H, $\left.\left(\mathrm{CH}_{2}\right)_{2}\right), 3.32-3.53\left(\mathrm{~m}, 4 \mathrm{H},\left(\mathrm{CH}_{2}\right)_{2}\right), 4.45\left(\mathrm{~s}, 2 \mathrm{H}, \mathrm{CH}_{2}\right), 7.08-7.62(\mathrm{~m}, 8 \mathrm{H}, \mathrm{Ar}-\mathrm{H}), 9.11$ (s, $1 \mathrm{H}, \mathrm{N}=\mathrm{CH}) .{ }^{13} \mathrm{C}-\mathrm{NMR}\left(\mathrm{CDCl}_{3}, 75 \mathrm{MHz}, \mathrm{ppm}\right): \delta 145.62,139.87,136.91,136.77,136.49,135.57,135.07$, $134.65,128.88,127.08,126.86,123.54,121.60,62.81,53.24,46.25,21.16 ; \mathrm{MS} m / z: 359(\mathrm{M}+1)$.

4-(4-(4-Methoxybenzyl)piperazin-1-yl)-[1,2,4]triazolo[4,3-a] quinoxaline (6i). Yield: 66\%; m.p. $154-156{ }^{\circ} \mathrm{C}$. IR ( $\mathrm{KBr}) \mathrm{cm}^{-1}: 1549,1513,1479(\mathrm{C}=\mathrm{N}) .{ }^{1} \mathrm{H}-\mathrm{NMR}\left(\mathrm{CDCl}_{3}, 300 \mathrm{MHz}, \mathrm{ppm}\right): 2.45-2.59\left(\mathrm{~m}, 4 \mathrm{H},\left(\mathrm{CH}_{2}\right)_{2}\right)$, 3.44-3.49 (m, 4H, $\left.\left(\mathrm{CH}_{2}\right)_{2}\right), 3.81\left(\mathrm{~s}, 2 \mathrm{H}, \mathrm{CH}_{2}\right), 4.41\left(\mathrm{~s}, 3 \mathrm{H}, \mathrm{CH}_{3}\right), 7.27-7.73(\mathrm{~m}, 8 \mathrm{H}, \mathrm{Ar}-\mathrm{H}), 9.17(\mathrm{~s}, 1 \mathrm{H}$, $\mathrm{N}=\mathrm{CH}) .{ }^{13} \mathrm{C}-\mathrm{NMR}\left(\mathrm{CDCl}_{3}, 75 \mathrm{MHz}, \mathrm{ppm}\right): \delta 158.82,145.61,139.87,136.88,135.55,130.43,129.63,127.83$, $126.83,123.57,121.56,114.48,113.54,62.41,55.25,53.12,46.16 ; \mathrm{MS} m / z: 375(\mathrm{M}+1)$.

4-(4-(2,4-Dichlorobenzyl)piperazin-1-yl)-[1,2,4]triazolo[4,3-a] quinoxaline (6j). Yield: 59\%; m.p. $180-182{ }^{\circ} \mathrm{C}$. IR (KBr) cm ${ }^{-1}: 1545,1508,1469(\mathrm{C}=\mathrm{N}) .{ }^{1} \mathrm{H}-\mathrm{NMR}\left(\mathrm{CDCl}_{3}, 300 \mathrm{MHz}, \mathrm{ppm}\right): 2.60-2.77\left(\mathrm{~m}, 4 \mathrm{H},\left(\mathrm{CH}_{2}\right)_{2}\right)$, 3.47-3.82 (m, 4H, $\left.\left(\mathrm{CH}_{2}\right)_{2}\right), 4.42\left(\mathrm{~s}, 2 \mathrm{H}, \mathrm{CH}_{2}\right), 7.14-7.72(\mathrm{~m}, 7 \mathrm{H}, \mathrm{Ar}-\mathrm{H}), 9.16(\mathrm{~s}, 1 \mathrm{H}, \mathrm{N}=\mathrm{CH}) .{ }^{13} \mathrm{C}-\mathrm{NMR}$ ( $\left.\mathrm{CDCl}_{3}, 75 \mathrm{MHz}, \mathrm{ppm}\right): \delta 145.58,139.70,136.65,135.67,134.82,134.45,133.17,132.74,131.77,131.63$, 128.90, 127.48, 126.92, 123.84, 121.90, 55.19, 53.85, 49.36; MS m/z: $413(\mathrm{M}+1)$.

4-(4-(2,6-Dichlorobenzyl)piperazin-1-yl)-[1,2,4]triazolo[4,3-a] quinoxaline (6k). Yield: 53\%; m.p. 198-200 ${ }^{\circ} \mathrm{C}$. IR (KBr) cm ${ }^{-1}: 1549,1510,1483(\mathrm{C}=\mathrm{N}) .{ }^{1} \mathrm{H}-\mathrm{NMR}\left(\mathrm{CDCl}_{3}, 300 \mathrm{MHz}, \mathrm{ppm}\right): 2.62-2.77\left(\mathrm{~m}, 4 \mathrm{H},\left(\mathrm{CH}_{2}\right)_{2}\right)$, 3.47-3.79 (m, 4H, $\left.\left(\mathrm{CH}_{2}\right)_{2}\right), 4.46\left(\mathrm{~s}, 2 \mathrm{H}, \mathrm{CH}_{2}\right), 7.21-7.70(\mathrm{~m}, 7 \mathrm{H}, \mathrm{Ar}-\mathrm{H}), 9.17(\mathrm{~s}, 1 \mathrm{H}, \mathrm{N}=\mathrm{CH}) .{ }^{13} \mathrm{C}-\mathrm{NMR}$ $\left(\mathrm{CDCl}_{3}, 75 \mathrm{MHz}, \mathrm{ppm}\right): \delta 145.62,139.99,137.04,135.50,133.99,130.94,129.00,128.83,128.43,127.95$, $123.63,121.60,114.48,56.45,53.20,50.83 ; \mathrm{MS} m / z: 413(\mathrm{M}+1)$. 
3.2.2. $[1,2,4]$ triazolo[4,3-a] quinoxaline Derivatives Bearing Substituted Benzoylpiperazine Moieties $(7 \mathbf{a}-\mathbf{e})$

A mixture of $5(0.20 \mathrm{~g}, 1.0 \mathrm{mmol})$, monosubstituted piperazine $(2.0 \mathrm{mmol})$ and anhydrous potassium carbonate in acetone was heated at reflux with stirring for $8 \mathrm{~h}$. The solvent was evaporated under reduced pressure, and the resulting residue dissolved in DCM. The DCM solution was washed sequentially with water and brine, dried over $\mathrm{MgSO}_{4}$, and distilled to dryness under reduced pressure. The resulting residue was purified by silica gel column chromatography with DCM and methanol (20:1). The yield, melting point, and spectral data of each compound were recorded.

(4-([1,2,4]Triazolo[4,3-a]quinoxalin-4-yl)piperazin-1-yl)(4-fluorophenyl)methanone (7a). Yield: 57\%; m.p.: 170-172 ${ }^{\circ} \mathrm{C}$; IR (KBr) cm ${ }^{-1}: 1638(\mathrm{C}=\mathrm{O}), 1540,1519,1459(\mathrm{C}=\mathrm{N}) ;{ }^{1} \mathrm{H}-\mathrm{NMR}\left(\mathrm{CDCl}_{3}, 300 \mathrm{MHz}, \mathrm{ppm}\right)$ : 8 3.59-3.66 (m, 4H, $\left.\left(\mathrm{CH}_{2}\right)_{2}\right), 3.81-4.37\left(\mathrm{~m}, 4 \mathrm{H},\left(\mathrm{CH}_{2}\right)_{2}\right), 7.01-7.68(\mathrm{~m}, 8 \mathrm{H}, \mathrm{Ar}-\mathrm{H}), 9.15(\mathrm{~s}, 1 \mathrm{H}, \mathrm{N}=\mathrm{CH})$. ${ }^{13} \mathrm{C}-\mathrm{NMR}\left(\mathrm{CDCl}_{3}, 75 \mathrm{MHz}, \mathrm{ppm}\right): \delta 169.72,165.18,160.69,145.49,139.64,137.28,136.13,131.36,128.05$, 127.11, 124.43, 121.75, 115.27, 114.70, 53.50, 50.55; MS m/z: 377 (M + 1).

(4-([1,2,4]Triazolo[4,3-a]quinoxalin-4-yl)piperazin-1-yl)(4-chlorophenyl)methanone (7b). Yield: 55\%; m.p.: 176-178 ${ }^{\circ} \mathrm{C}$; IR (KBr) cm ${ }^{-1}: 1630$ (C=O), 1544, 1516, $1457(\mathrm{C}=\mathrm{N}) ;{ }^{1} \mathrm{H}-\mathrm{NMR}\left(\mathrm{CDCl}_{3}, 300 \mathrm{MHz}, \mathrm{ppm}\right)$ : 8 3.71-3.98 (m, 4H, $\left.\left(\mathrm{CH}_{2}\right)_{2}\right), 4.50-5.30\left(\mathrm{~m}, 4 \mathrm{H},\left(\mathrm{CH}_{2}\right)_{2}\right), 7.27-7.78(\mathrm{~m}, 8 \mathrm{H}, \mathrm{Ar}-\mathrm{H}), 9.22(\mathrm{~s}, 1 \mathrm{H}, \mathrm{N}=\mathrm{CH})$. ${ }^{13} \mathrm{C}-\mathrm{NMR}\left(\mathrm{CDCl}_{3}, 75 \mathrm{MHz}, \mathrm{ppm}\right): \delta 169.59,145.55,139.75,136.36,136.18,135.96,133.67,128.93,128.74$, $128.18,127.21,124.57,121.79,114.64,56.04,50.55 ; \mathrm{MS} \mathrm{m} / z: 393(\mathrm{M}+1)$.

(4-([1,2,4]Triazolo[4,3-a]quinoxalin-4-yl)piperazin-1-yl)(phenyl)methanone (7c). Yield: 76\%; m.p.: 216-218 ${ }^{\circ} \mathrm{C}$; IR (KBr) cm ${ }^{-1}: 1625(\mathrm{C}=\mathrm{O}), 1544,1508,1460(\mathrm{C}=\mathrm{N}) ;{ }^{1} \mathrm{H}-\mathrm{NMR}\left(\mathrm{CDCl}_{3}, 300 \mathrm{MHz}, \mathrm{ppm}\right): \delta$ 3.51-3.69 $\left(\mathrm{m}, 4 \mathrm{H},\left(\mathrm{CH}_{2}\right)_{2}\right), 3.99-4.49\left(\mathrm{~m}, 4 \mathrm{H},\left(\mathrm{CH}_{2}\right)_{2}\right), 7.09-7.76(\mathrm{~m}, 9 \mathrm{H}, \mathrm{Ar}-\mathrm{H}), 9.20(\mathrm{~s}, 1 \mathrm{H}, \mathrm{N}=\mathrm{CH}) .{ }^{13} \mathrm{C}-\mathrm{NMR}$ $\left(\mathrm{CDCl}_{3}, 75 \mathrm{MHz}\right.$, ppm): $\delta 170.68,146.25,139.78,137.62,135.67,135.39,130.04,129.54,128.64,127.23$, $127.14,124.45,121.80,114.63,52.74,48.83 ; \mathrm{MS} m / z: 359(\mathrm{M}+1)$.

(4-([1,2,4]Triazolo[4,3-a]quinoxalin-4-yl)piperazin-1-yl)(p-tolyl)methanone (7d). Yield: 71\%; m.p.: $168-170{ }^{\circ} \mathrm{C}$; IR $(\mathrm{KBr}) \mathrm{cm}^{-1}: 1624(\mathrm{C}=\mathrm{O}), 1544,1516,1458(\mathrm{C}=\mathrm{N}) ;{ }^{1} \mathrm{H}-\mathrm{NMR}\left(\mathrm{CDCl}_{3}, 300 \mathrm{MHz}, \mathrm{ppm}\right): \delta 2.41$ (s, $3 \mathrm{H}, \mathrm{CH} 3), 3.48-3.53\left(\mathrm{~m}, 4 \mathrm{H},\left(\mathrm{CH}_{2}\right)_{2}\right), 3.76-3.95\left(\mathrm{~m}, 4 \mathrm{H},\left(\mathrm{CH}_{2}\right)_{2}\right), 7.23-7.71(\mathrm{~m}, 8 \mathrm{H}, \mathrm{Ar}-\mathrm{H}), 9.21(\mathrm{~s}, 1 \mathrm{H}$, $\mathrm{N}=\mathrm{CH}) .{ }^{13} \mathrm{C}-\mathrm{NMR}\left(\mathrm{CDCl}_{3}, 75 \mathrm{MHz}, \mathrm{ppm}\right): \delta 169.99,147.11,141.30,137.43,136.74,135.43,133.67,128.94$, $128.37,127.39,126.21,124.52,121.94,114.51,52.06,49.73,21.57$; MS m/z: $373(\mathrm{M}+1)$.

(4-([1,2,4]Triazolo[4,3-a]quinoxalin-4-yl)piperazin-1-yl)(4-methoxyphenyl)methanone (7e). Yield: 59\%; m.p.: 198-200 ${ }^{\circ} \mathrm{C}$; IR (KBr) cm ${ }^{-1}: 1624$ (C=O), 1545, 1516, 1460 (C=N) ; ${ }^{1} \mathrm{H}-\mathrm{NMR}\left(\mathrm{CDCl}_{3}, 300 \mathrm{MHz}, \mathrm{ppm}\right)$ : 反 3.43-3.51 (m, 4H, $\left.\left(\mathrm{CH}_{2}\right)_{2}\right), 3.67-3.86\left(\mathrm{~m}, 4 \mathrm{H},\left(\mathrm{CH}_{2}\right)_{2}\right), 3.97\left(\mathrm{~s}, 3 \mathrm{H}, \mathrm{CH}_{3}\right), 7.21-7.70(\mathrm{~m}, 8 \mathrm{H}, \mathrm{Ar}-\mathrm{H}), 9.19$ (s, $1 \mathrm{H}, \mathrm{N}=\mathrm{CH}) .{ }^{13} \mathrm{C}-\mathrm{NMR}\left(\mathrm{CDCl}_{3}, 75 \mathrm{MHz}, \mathrm{ppm}\right): \delta 170.14,149.27,143.30,139.49,137.44,136.79,134.44$, $129.90,128.00,127.94,126.39,124.82,121.04,114.73,60.21,53.61,47.93 ; \mathrm{MS} m / z: 377(\mathrm{M}+1)$.

\subsection{Pharmacology}

The method of measuring stroke volume in the left atrium (LA) has been described [13]. The features of CHF are cardiac dilatation, poor contractility of cardiac muscle, decreased ejection fraction, and depression of left ventricular pressure maximum alleosis. Therefore, macroscopic measurement of the variance in LA stroke volume can be used to estimate the positive inotropic effects of the compounds synthesized. Milrinone (Shuzhou Unite Pharmaceuticals, Shuzhou, China), dimethyl sulfoxide (DMSO; Sigma-Aldrich) were purchased. All other reagents were of analytical grade. Atria were obtained from New Zealand white rabbits, and the mean weight of the LA was $183.6 \pm 6.8 \mathrm{mg}$. Hearts were removed from rabbits and the LA dissected free. A calibrated transparent atrial cannula containing two small catheters was inserted into the LA. The cannulated LA was transferred to an organ chamber and perfused immediately with $\mathrm{N}$-2-hydroxyethyl piperazine- $\mathrm{N}$-2-ethanesulfonic acid (HEPES) buffer solution by means of a peristaltic pump $(1.25 \mathrm{~mL} / \mathrm{min})$ at $34{ }^{\circ} \mathrm{C}$. The composition of the buffer was (in mM): $118 \mathrm{NaCl}, 4.7 \mathrm{KCl}, 2.5 \mathrm{CaCl}_{2}, 1.2 \mathrm{MgCl}_{2}, 25 \mathrm{NaHCO}_{3}, 10.0$ glucose, 10.0 HEPES (adjusted to $\mathrm{pH} 7.4$ with $1 \mathrm{M} \mathrm{NaOH}$ ) and $0.1 \%$ bovine serum albumin. Soon after the perfusion system 
was set up, transmural electrical field stimulation with a luminal electrode was started at $1.5 \mathrm{~Hz}$ (duration, $0.3-0.5 \mathrm{~ms}$; voltage, $30 \mathrm{~V}$ ). Changes in LA stroke volume were monitored by reading the lowest level of the water column in the calibrated atrial cannula during end diastole. Atria were perfused for $60 \mathrm{~min}$ to stabilize the stroke volume. The atrial beat rate was fixed at $1.5 \mathrm{~Hz}, \mathrm{LA}$ stroke volume recorded at $2 \mathrm{~min}$ intervals, and the stimulus effect of the sample recorded after one circulation in the control group. Each circulation was $12 \mathrm{~min}$. Compounds were investigated using the single-dose method at $3 \times 10^{-5} \mathrm{M}$. Samples were dissolved in DMSO and diluted with HEPES buffer to $0.1 \%$ DMSO. Biological data for these compounds were expressed in mean percentage values of increased stroke volume (Table 1). Heart-rate measurements for selected compounds were carried out in isolated rabbit hearts by recording the electrocardiogram in the volume conduction model. To assess differences, repeated measurements were compared by an ANOVA test followed by Bonferroni's multiple-comparison test. $p<0.05$ was considered significant and data are the mean $\pm \mathrm{SE}$.

\section{Conclusions}

Two series of $[1,2,4]$ triazolo[4,3-a] quinoxaline derivatives bearing substituted benzylpiperazine and benzoylpiperazine moieties were synthesized using $\mathbf{A}$ as the lead compound. We tried to ascertain potent compounds for cardiac contractility without increasing the heart rate. Compound $\mathbf{6 c}$ exhibited promising cardiovascular properties and potent activities compared with milrinone: $6 \mathbf{c}$ was 5.1 -fold more active than milrinone. This compound is undergoing further biological tests, including in vivo evaluation, coronary vasodilation tests, and studies into possible mechanisms of action.

Acknowledgments: This work was supported by the National Science Foundation of China (81160381).

Author Contributions: Xue-Kun Liu and Hu-Ri Piao conceived and designed the study; Long-Xu Ma and Zhi-Yu Wei performed the synthetic experiments; Xun Cui, Shi Zhan and Xiu-Mei Yin performed the biological evaluation experiments. All authors read and approved the final manuscript.

Conflicts of Interest: The authors declare no conflict of interest.

\section{References}

1. The Digitalis Investigation Group. The effect of digoxin on mortality and morbidity in patients with heart failure. N. Engl. J. Med. 1997, 336, 525-533.

2. Fujioka, T.; Teramoto, S.; Mori, T.; Hosokawa, T.; Sumida, T.; Tominaga, M.; Yabuuchi, Y. Novel positive inotropic agents: Synthesis and biological activities of 6-(3-amino-2-hydroxypropoxy)-2(1H)-quinolinone derivatives. J. Med. Chem. 1992, 35, 3607-3612. [CrossRef] [PubMed]

3. Packer, M.; Carver, J.R.; Rodeheffer, R.J.; Ivanhoe, R.J.; DiBianco, R.; Zeldis, S.M.; Hendrix, G.H.; Bommer, W.J.; Elkayam, U.; Kukin, M.L.; et al. Effect of oral milrinone on mortality in severe chronic heart failure. N. Engl. J. Med. 1991, 325, 1468-1475. [CrossRef] [PubMed]

4. Cohn, J.N.; Oldstein, S.O.; Greenberg, B.H.; Lorell, B.H.; Bourge, R.C.; Jaski, B.E.; Gottlieb, S.O.; McGrew, F.D.; DeMets, L.; White, B.G. A dose-dependent increase in mortality with vesnarinone among patients with severe heart failure. Vesnarinone Trial Investigators. N. Engl. J. Med. 1998, 339, 1810-1816. [CrossRef] [PubMed]

5. Harada, K.; Supriatno, E.; Yoshida, H.; Sato, M. Vesnarinone inhibits angiogenesis and tumorigenicity of human oral squamous cell carcinoma cells by suppressing the expression of vascular endothelial growth factor and interleukin-8. Int. J. Oncol. 2005, 27, 1489-1497. [PubMed]

6. Neubauer, S. The failing heart-An engine out of fuel. N. Engl. J. Med. 2007, 356, 1140-1151. [CrossRef] [PubMed]

7. Wu, Y.; Ma, L.X.; Che, J.; Sun, L.P.; Song, M.X.; Cui, X.; Piao, H.R. Synthesis and biological evaluation of $[1,2,4]$ triazolo[3,4-a]phthalazine and tetrazolo[5,1-a]phthalazine derivatives bearing substituted benzylpiperazine moieties as positive inotropic Agents. Chem. Biol. Drug. Des. 2013, 81, 591-599. [CrossRef] [PubMed]

8. Chen, H.; Zhao, X.Y.; Bai, M.; Ning, D.B.; Gong, P.; Han, J. Synthesis of XL765 as a phosphoinositide-3-kinase (PI3K) inhibitor. Chin. J. Med. Chem. 2011, 21, 290-294. 
9. Ubarhande, S.S.; Devhate, P.P.; Berad, B.N. Green synthesis of quinoxaline and substituted quinoxalines. Int. J. Chem. Sci. 2011, 9, 1768-1774.

10. Ajani, O.O.; Obafemi, C.A.; Nwinyi, O.C.; Akinpelu, D.A. Microwave assisted synthesis and antimicrobial activity of 2-quinoxalinone-3-hydrazone derivatives. Bioorg. Med. Chem. 2010, 18, 214-221. [CrossRef] [PubMed]

11. Kshirsagar, S.S.; Nimje, H.M.; Chaudhari, P.S.; Bayas, J.P.; Patel, E.K.; Karjikar, A.H.; Girme, P.D.; Oswal, R.J. Synthesis and Antimicrobial Activity of Novel Thiazolidinone and Azetidinone Derivatives. Asian J. Chem. 2011, 23, 4021-4023.

12. Galal, S.A.; Abdelsamie, A.S.; Soliman, S.M.; Mortier, J.; Wolber, G.; Ali, M.M.; Tokuda, H.; Suzuki, N.; Lida, A.; Ramadan, R.A. Design, synthesis and structure-activity relationship of novel quinoxaline derivatives as cancer chemopreventive agent by inhibition of tyrosine kinase receptor. Eur. J. Med. Chem. 2013, 69, 115-124. [CrossRef] [PubMed]

13. Okada, R.; Negishi, N.; Nagaya, H. Design, The role of the nigrotegmental GABAergic pathway in the propagation of pentylenetetrazol-induced seizures. Brain Res. 1989, 480, 383-387. [CrossRef]

Sample Availability: Not Available.

(C) 2017 by the authors; licensee MDPI, Basel, Switzerland. This article is an open access article distributed under the terms and conditions of the Creative Commons Attribution (CC BY) license (http:/ / creativecommons.org/licenses/by/4.0/). 